\title{
PRELIMIT AND LIMIT GENERALIZATIONS OF THE POLLACZEK-KHINCHIN FORMULA
}

UDC 519.21

\author{
D. V. GUSAK
}

ABSTRACT. The moment generating function of the nondegenerate distribution of the maximum $\xi^{+}=\sup _{0 \leq t<\infty} \xi(t)$ of a compound Poisson process

$$
\xi(t)=a t+S(t), \quad a<0, \quad S(t)=\sum_{k \leq \nu(t)} \xi_{k}, \quad \xi_{k}>0,
$$

where $\nu(t)$ is a simple Poisson process with intensity $\lambda>0$, is determined via the well-known Pollaczek-Khinchin formula if $m=\mathrm{E} \xi(1)<0$.

We obtain a prelimit generalization of this formula that determines the LaplaceCarson transform of the moment generating function of the maximum $\xi^{+}(t)=$ $\sup _{0 \leq t^{\prime} \leq t} \xi\left(t^{\prime}\right), 0<t<\infty$, and the moment generating function of $\xi^{+}=\xi^{+}(\infty)$ under the assumption that $m<0$ for homogeneous processes $\xi(t)$ with independent increments and of bounded variation. Relationships of a different type between characteristic functions of $\xi^{+}\left(\theta_{s}\right)\left(\mathrm{P}\left\{\theta_{s}>t\right\}=e^{-s t}, s, t>0\right)$ and of $\xi^{+}$are also obtained by using earlier results presented by the author.

\section{INTRODUCTION}

Let $\xi(t)$ be a lower continuous compound Poisson process whose characteristic function is defined by the cumulant $\psi(\alpha)$, that is,

$$
\begin{gathered}
\mathrm{E} e^{i \alpha \xi(t)}=e^{t \psi(\alpha)}, \quad t \geq 0, \quad \psi(\alpha)=i \alpha a+\lambda(\varphi(\alpha)-1), \\
a<0, \quad \lambda>0, \quad \varphi(\alpha)=\mathrm{E} e^{i \alpha \xi_{k}}, \\
F(x)=\mathrm{P}\left\{\xi_{k}<x\right\}, \quad x \geq 0, \quad \bar{F}(x)=1-F(x) .
\end{gathered}
$$

If

$$
m=\mathrm{E} \xi(1)=a+\lambda \mu_{1}<0, \quad \mu_{1}=\mathrm{E} \xi_{k}, \quad F(0)=0,
$$

then the moment generating function of $\xi^{+}=\sup _{0 \leq t<\infty} \xi(t)$ is determined via the Pollaczek-Khinchin formula

$$
\begin{gathered}
\mathrm{E} e^{-z \xi^{+}}=\frac{p_{+}}{1-q_{+} \widetilde{F}(z) / \mu_{1}}, \quad p_{+}=\mathrm{P}\left\{\xi^{+}=0\right\}=1-q_{+}, \\
\widetilde{F}(z)=\int_{0}^{\infty} \bar{F}(x) e^{-z x} d x .
\end{gathered}
$$

This result is often used in queueing theory and in risk theory (see, for example, [1]-[3]).

2000 Mathematics Subject Classification. Primary 60G50; Secondary 60K10.

Key words and phrases. Semicontinuous compound Poisson processes, semicontinuous homogeneous processes with independent increments, Pollaczek-Khinchin formula and its generalizations. 
First we consider the case of a process $\xi(t)$ with bounded variation if its cumulant is given by

$$
\psi(\alpha)=i \alpha a+\int_{-\infty}^{\infty}\left(e^{i \alpha x}-1\right) \Pi(d x), \quad \int_{|x| \leq 1}|x| \Pi(d x)<\infty, \quad a \leq 0 .
$$

Let

$$
\begin{gathered}
\xi^{ \pm}(t)=\underset{0 \leq t^{\prime} \leq t}{\sup (\inf ) \xi\left(t^{\prime}\right),} \quad \xi^{ \pm}=\underset{0 \leq t<\infty}{\sup (\inf )} \xi(t), \\
\tau^{+}(x)=\inf \{t>0: \xi(t)>x\}, \quad \gamma^{+}(x)=\xi\left(\tau^{+}(x)\right)-t, \quad x \geq 0 .
\end{gathered}
$$

If $\int_{-\infty}^{0} \Pi(d x)=0$, then $a<0$. Assume that

$$
\mathrm{P}\left\{\tau^{+}(0)=\gamma^{+}(0)=0\right\}=0 .
$$

We prove a generalization of formula (1) for the processes $\xi(t)$ satisfying the latter condition and whose cumulant is given by (2).

The moment Generating Functions of $\xi^{+}\left(\theta_{s}\right)$ And $\xi^{+}$IN The CAse of $m<0$

Let $\theta_{s}$ be an exponential random variable with parameter $s>0$ and let $\theta_{s}$ be independent of $\xi(t)$. Then the Laplace-Carson transforms of the characteristic functions of $\xi(t)$ and $\xi^{ \pm}(t)$ are given by

$$
\begin{gathered}
\varphi(s, \alpha)=s \int_{0}^{\infty} e^{-s t} \mathrm{E} e^{i \alpha \xi(t)} d t=\frac{s}{s-\psi(\alpha)} \\
\varphi_{ \pm}(s, \alpha)=s \int_{0}^{\infty} \mathrm{E} e^{i \alpha \xi^{ \pm}(t)} e^{-s t} d t=\mathrm{E} e^{i \alpha \xi^{ \pm}\left(\theta_{s}\right)}
\end{gathered}
$$

The joint moment generating function of $\left\{\tau^{+}(0), \gamma^{+}(0)\right\}$ is given by

$$
\begin{gathered}
f_{+}(s, z)=\mathrm{E}\left[e^{-z \gamma^{+}(0)-s \tau^{+}(0)}, \tau^{+}(0)<\infty\right]=\mathrm{E}\left[e^{-z \gamma^{+}(0)}, \xi^{+}\left(\theta_{s}\right)>0\right], \\
f_{+}(s, z) \underset{z \rightarrow 0}{\rightarrow} \mathrm{P}\left\{\xi^{+}\left(\theta_{s}\right)>0\right\}=q_{+}(s), \quad p_{+}(s)=1-q_{+}(s)>0
\end{gathered}
$$

if assumption (3) holds.

Theorem 1. Let $\xi(t)$ be a homogeneous process with independent increments and with bounded variation (its cumulant is given by (2)). Under condition (3) the prelimit Pollaczek-Khinchin formula determines the moment generating function

$$
\mathrm{E} e^{-z \xi^{+}\left(\theta_{s}\right)}=: \varphi_{+}(s, i z)=\frac{p_{+}(s)}{1-q_{+}(s) \mathrm{E}\left[e^{-z \gamma^{+}(0)} / \xi^{+}\left(\theta_{s}\right)>0\right]}
$$

for $s>0$.

If $m=\mathrm{E} \xi(1)<0$, then the moment generating function of $\xi^{+}$is obtained by passing to the limit as $s \rightarrow 0$ in the Pollaczek-Khinchin formula, namely

$$
\mathrm{E} e^{-z \xi^{+}}=\frac{p_{+}}{1-q_{+} \mathrm{E}\left[e^{-z \gamma^{+}(0)} / \xi^{+}>0\right]}, \quad p_{+}=\mathrm{P}\left\{\xi^{+}=0\right\}>0 .
$$

Proof. Let $\theta_{\mu}^{\prime}$ be an exponential random variable that is independent of $\theta_{s}$ and $\xi(t)$. Then the second factorization equality (see equality (2.26) in [4]) implies that

$$
\begin{gathered}
\mathrm{E}\left[e^{-s \tau^{+}\left(\theta_{\mu}^{\prime}\right)-z \gamma^{+}\left(\theta_{\mu}^{\prime}\right)}, \tau^{+}\left(\theta_{\mu}^{\prime}\right)<\infty\right]=\frac{\mu}{\mu-z}\left\{1-\frac{\varphi_{+}(s, i \mu)}{\varphi_{+}(s, i z)}\right\}, \\
s>0, \quad z>0, \quad \mu>0 .
\end{gathered}
$$


Then

$$
\begin{aligned}
f_{+}(s, z) & =\mathrm{E}\left[e^{-z \gamma^{+}(0)-s \tau^{+}(0)}, \tau^{+}(0)<0\right]=\mathrm{E}\left[e^{-z \gamma^{+}(0)}, \xi^{+}\left(\theta_{s}\right)>0\right] \\
& =1-\frac{p_{+}(s)}{\varphi_{+}(s, i z)}
\end{aligned}
$$

as $\mu \rightarrow \infty$ in view of relation (2.29) in [4]. Equality (9) implies that

$$
\varphi_{+}(s, i z)=\frac{p_{+}(s)}{1-f_{+}(s, z)}=\frac{p_{+}(s)}{1-q_{+}(s) \widetilde{g}_{s}(z)},
$$

where $\widetilde{g}_{s}(z)=\mathrm{E}\left[e^{-z \gamma^{+}(0)} / \xi^{+}\left(\theta_{s}\right)>0\right]$. This proves formula (6).

If $m<0$, then passing to the limit as $s \rightarrow 0$ we obtain equality (7) with the conditional moment generating function

$$
\widetilde{g}_{0}(z)=\mathrm{E}\left[e^{-z \gamma^{+}(0)} / \xi^{+}>0\right] .
$$

EVAluAtion OF $\widetilde{g}_{s}(z)$ AND $\widetilde{g}_{0}(z)$ FOR LOWER CONTINUOUS PROCESSES $\xi(t)$ AND DERIVATION OF THE MOMENT GENERATING FUNCTIONS FOR $\xi^{+}\left(\theta_{s}\right)$ AND $\xi^{+}$

A lower continuous process $\xi(t)$ having a bounded variation and satisfying condition (3) is determined by the moment generating function

$$
\psi(\alpha)=i \alpha a+\int_{0}^{\infty}\left(e^{i \alpha x}-1\right) \Pi(d x), \quad \int_{0}^{1} x \Pi(d x)<\infty, a<0 .
$$

If $\int_{0}^{\infty} \Pi(d x)=\lambda<\infty$, then $\Pi(d x)=\lambda d F(x), x>0, \xi(t)=a t+S(t)$, and

$$
\psi(\alpha)=i \alpha a+\lambda \int_{0}^{\infty}\left(e^{i \alpha x}-1\right) d F(x)=i \alpha a+\psi_{1}(\alpha),
$$

$$
\Pi(x)=\int_{x}^{\infty} \Pi(d y)=\lambda \bar{F}(x), \quad x>0, \quad \psi_{1}(\alpha)=\ln \mathrm{E} e^{i \alpha S(1)}=i \alpha \lambda \int_{0}^{\infty} \bar{F}(x) e^{i \alpha x} d x .
$$

According to Theorem 3.2 of [4], the Lundberg equation

$$
k(r)=\psi(-i r)=s
$$

possesses a negative root $r_{s}=-\rho_{-}(s)<0$ and $\rho_{-}(s)$ determines the characteristic function of $\xi^{-}\left(\theta_{s}\right)$ as follows:

$$
\varphi_{-}(s, \alpha)=\mathrm{E} e^{i \alpha \xi^{-}\left(\theta_{s}\right)}=\frac{\rho_{-}(s)}{\rho_{-}(s)+i \alpha}, \quad p_{+}(s)=\frac{s}{|a| \rho_{-}(s)} .
$$

For lower continuous processes, the function $k(r)$ is convex for $r<r_{+}$,

$$
0 \leq r_{+}=\sup \{r: k(r)<\infty\} .
$$

Moreover the function $s-k(r)$ has at most two roots in view of the results of $\S 11$ in [1]. The minimal of those roots $r_{s}=-\rho_{-}(s)$ (defined if $s \geq \inf k(r)$ ) is a root of equation (13) and determines the function $\varphi_{-}(s, \alpha)$. The root $r_{s}$ has the following properties: $\rho_{-}(s)>0$ for $s>0$; if $m=\mathrm{E} \xi(1)=k^{\prime}(0) \leq 0$, then

$$
\rho_{-}(s) \rightarrow 0 \text { as } s \rightarrow 0 .
$$

Similarly, the positive root

$$
r_{s}=\rho_{+}(s)>0
$$

of equation (13) determines $\varphi_{+}(s, \alpha)$ for upper continuous processes. In a certain sense, the random function

$$
T(x)=\tau^{+}(x), \quad x \geq 0,
$$


is inverse to a nondecreasing process $y=\xi^{+}(t)$ and thus $T(x)=\tau^{+}(x)$ also is nondecreasing and has a bounded variation. By formula (23.2) of [6, $\S 23],-\rho_{+}(s)$ is the cumulant of $T(x)$ and thus, by Theorem 2 of $[6, \S 17]$ it can be written down as follows:

$$
-\rho_{+}(s)=\ln \mathrm{E} e^{-s T(1)}=-\gamma_{+} s+\int_{0}^{\infty}\left(e^{-s y}-1\right) d N(y), \quad \gamma_{+}>0 .
$$

For both cases, the jumps of $T(x)=\tau^{ \pm}( \pm x)$ are determined by the intervals where the processes $\xi^{ \pm}(t)$ remain constant, while the intervals where $T(x)$ is growing with respect to $x$ are determined by the intervals where $\xi^{ \pm}(t)$ is linearly changing with respect to $t$.

In the case of the lower continuity, $r_{s}=-\rho_{-}(s)$ is the cumulant of $T(x)=\tau^{-}(-x)$, $x \geq 0$, and has a representation similar to that of $\rho_{+}(s)$ but with another constant $\gamma_{-}>0$. The function $N(y)$ determines the Lévy measure of jumps $\tau^{ \pm}( \pm x)$, namely $\Pi(d y)=d N(y)$. For example, in the case of a Wiener process $W(t)$ with $\mathrm{E} W(t)=0$, the processes $T(x)=\tau^{ \pm}( \pm x)$ are stable with respect to $x \geq 0$. The processes $T(x)$ are of index $\alpha_{*}=1 / 2$ and their cumulants have the following characteristics:

$$
\gamma_{ \pm}=0, \quad N(y)=-\sqrt{\frac{2}{\pi}} y^{-1 / 2}, \quad \Pi(d y)=\frac{1}{\sqrt{2 \pi}} y^{-3 / 2} d y, \quad y>0
$$

(see Theorem 2.10 in $[4, \S 2.5]$ ).

By Corollary 5.2 in [4, $\S 5.1]$,

$$
\widetilde{g}_{s}(z)=\frac{z \widetilde{\Pi}(z)-\rho_{-}(s) \widetilde{\Pi}\left(\rho_{-}(s)\right)}{\left(z-\rho_{-}(s)\right) \widetilde{\Pi}\left(\rho_{-}(s)\right)}, \quad \widetilde{\Pi}(z)=\int_{0}^{\infty} e^{-z x} \Pi(x) d x,
$$

for lower continuous processes. If $m=\mathrm{E} \xi(1)=k^{\prime}(0)<0$,

$$
\rho_{-}(s) \underset{s \rightarrow 0}{\rightarrow} 0 .
$$

One can evaluate the conditional moment generating function of $\gamma^{+}(0)$ in this case by passing to the limit as follows:

$$
\widetilde{g}_{0}(z)=\lim _{s \rightarrow 0} \tilde{g}_{s}(z)=\mathrm{E}\left[e^{-z \gamma^{+}(0)} / \zeta^{+}>0\right]=\frac{\widetilde{\Pi}(z)}{\widetilde{\Pi}(0)} .
$$

If $\lambda=\Pi(0)<\infty$, then $\widetilde{\Pi}(z)=\lambda \widetilde{F}(z)=\lambda \int_{0}^{\infty} e^{-z x} \bar{F}(x) d x$ and

$$
\widetilde{g}_{0}(z)=\frac{\widetilde{F}(z)}{\widetilde{F}(0)}, \quad \widetilde{F}(0)=\int_{0}^{\infty} \bar{F}(x) d x=\mu_{1} .
$$

The following result follows from Theorem 1 and the latter reasoning.

Corollary 1. Let $\xi(t)$ be a lower semicontinuous process and let its cumulant be given by (11). Then the following prelimit generalization of the Pollaczek-Khinchin formula holds:

$$
\varphi_{+}(s, i z)=\frac{p_{+}(s)}{1-q_{+}(s) \frac{z \widetilde{\Pi}(z)-\rho_{-}(s) \widetilde{\Pi}\left(\rho_{-}(s)\right)}{\left(z-\rho_{-}(s)\right) \widetilde{\Pi}\left(\rho_{-}(s)\right)}}, \quad q_{+}(s)=\frac{1}{|a|} \widetilde{\Pi}\left(\rho_{-}(s)\right) .
$$

If $\lambda=\Pi(0)<\infty$ (that is, in the case of processes $\xi(t)$ whose cumulant is given by equality (12)), then

$$
\varphi_{+}(s, i z)=\frac{p_{+}(s)}{1-q_{+}(s) \frac{z \widetilde{F}(z)-\rho_{-}(s) \tilde{F}\left(\rho_{-}(s)\right)}{\left(z-\rho_{-}(s)\right) \tilde{F}\left(\rho_{-}(s)\right)}}, \quad q_{+}(s)=\frac{\lambda \widetilde{F}\left(\rho_{-}(s)\right)}{|a|} .
$$


If $m=\mathrm{E} \xi(1)<0$, then passing to the limit as $s \rightarrow 0$ in equalities (18) and (19) we obtain the following limit relations:

$$
\begin{gathered}
\mathrm{E} e^{-z \xi^{+}}=\frac{p_{+}}{1-q_{+} \widetilde{\Pi}(z) / \widetilde{\Pi}(0)}, \quad q_{+}=\frac{1}{|a|} \widetilde{\Pi}(0), \\
\mathrm{E} e^{-z \xi^{+}}=\frac{p_{+}}{1-q_{+} \widetilde{F}(z) / \widetilde{F}(0)}, \quad q_{+}=\frac{\lambda}{|a|} \widetilde{F}(0)=\frac{\lambda \mu_{1}}{|a|}
\end{gathered}
$$

(the corresponding conditional moment generating functions for $\gamma^{+}(0)$ are given by equalities (16) and (17)). Equality (21) coincides with the classical Pollaczek-Khinchin formula.

Proof. Relations (19)-(21) follow from equalities (6)-(7) by using the expression for the values of $\widetilde{g}_{s}(z)$ given by $(15)$ and that for $\widetilde{g}_{0}(z)$ given by $(16)-(17)$.

Evaluation of the Joint moment Generating FunCtion of $\left\{\tau^{+}(0), \gamma^{+}(0)\right\}$ AND MOMENT GENERATING FUNCTIONS OF $\xi^{+}\left(\theta_{s}\right)$ AND $\xi^{+}$ FOR ALMOST LOWER SEMICONTINUOUS STEP PROCESSES

An almost lower semicontinuous step process is defined by

$$
\begin{gathered}
\xi(t)=\xi_{1}(t)+\xi_{2}(t), \quad \xi_{1,2}(t)=\sum_{k \leq \nu_{1,2}(t)} \xi_{k}^{\prime}\left(\xi_{k}^{\prime \prime}\right), \\
F_{1}(x)=\mathrm{P}\left\{\xi_{k}^{\prime}<x\right\}, \quad x>0, \quad F_{2}(x)=\mathrm{P}\left\{\xi_{k}^{\prime \prime}<x\right\}=e^{b x}, \quad b>0, x<0,
\end{gathered}
$$

where $\xi_{1,2}(t)$ are independent processes, $\nu_{1,2}(t)$ are simple independent Poisson processes with intensities $\lambda_{1,2}>0$,

$$
\begin{gathered}
\psi(\alpha)=\ln \mathrm{E} e^{i \alpha \xi(t)}=\psi_{1}(\alpha)+\psi_{2}(\alpha), \\
\psi_{1}(\alpha)=\lambda_{1} \int_{0}^{\infty}\left(e^{i \alpha x}-1\right) d F(x)=i \alpha \lambda_{1} \int_{0}^{\infty} e^{i \alpha x} \bar{F}(x) d x, \\
\psi_{2}(\alpha)=\lambda_{2} \int_{-\infty}^{0}\left(e^{i \alpha x}-1\right) b e^{b x} d x=-\frac{i \alpha \lambda_{2}}{b+i \alpha}, \quad \lambda_{2}>0, b>0 .
\end{gathered}
$$

By Lemma 3.4 in $4, \S 5.2$, if $\xi(t)$ is a process whose cumulant is given by (22), then the root

$$
r_{s}=-\rho_{-}(s)=-b p_{-}(s)<0
$$

of equation (13) determines the characteristic function of $\xi^{-}\left(\theta_{s}\right)$ as follows:

$$
\varphi_{-}(s, \alpha)=\frac{b\left(p_{-}(s)+i \alpha\right)}{\rho_{-}(s)+i \alpha}, \quad p_{+}(s) p_{-}(s)=\frac{s}{s+\lambda} .
$$

By Theorem 5.9 in $4, \S 5.2]$,

$$
\begin{aligned}
\int_{0}^{\infty} e^{-z y} \mathrm{P}\left\{\gamma^{+}(0)>y, \xi^{+}\left(\theta_{s}\right)>0\right\} d y \\
\quad=\frac{1}{s+\lambda}\left[\widetilde{\Pi}(z)+b q_{-}(s) \frac{\widetilde{\Pi}(z)-\widetilde{\Pi}\left(\rho_{-}(s)\right)}{\rho_{-}(s)-z}\right], \quad \widetilde{\Pi}(z)=\lambda \widetilde{F}(z) .
\end{aligned}
$$


Corollary 2. If $\xi(t)$ is an almost lower semicontinuous step process whose cumulant is given by (23), then the joint moment generating function of $\left\{\tau^{+}(0), \gamma^{+}(0)\right\}$ is equal to

$$
\begin{aligned}
& f_{+}(s, z)=\mathrm{E}\left[e^{-z \gamma^{+}(0)}, \xi^{+}\left(\theta_{s}\right)>0\right] \\
&=\frac{\lambda_{1}}{s+\lambda}\left[\varphi_{1}(i z)+b q_{-}(s) \frac{z \widetilde{F}_{1}(z)-\rho_{-}(s) \widetilde{F}_{1}\left(\rho_{-}(s)\right)}{z-\rho_{-}(s)}\right], \\
& q_{+}(s)=\frac{\lambda_{1}}{s+\lambda}\left[1+b q_{-}(s) \widetilde{F}_{1}\left(\rho_{-}(s)\right)\right], \quad \varphi_{1}(i z)=\int_{0}^{\infty} e^{-z x} d F_{1}(x),
\end{aligned}
$$

for $s>0$ where $\lambda=\lambda_{1}+\lambda_{2}$. If $m<0$, then

$$
\rho_{-}(s) \rightarrow 0, \quad q_{-}(s) \rightarrow 1
$$

as $s \rightarrow 0$ and, as a result,

$$
\begin{gathered}
f_{+}(0, z)=\mathrm{E}\left[e^{-z \gamma^{+}(0)}, \xi^{+}>0\right]=\frac{\lambda_{1}}{\lambda}\left[\varphi_{1}(i z)+b \widetilde{F}_{1}(z)\right], \\
q_{+}=\frac{\lambda_{1}}{\lambda}\left[1+b \widetilde{F}_{1}(0)\right], \quad \widetilde{F}_{1}(0)=\mathrm{E} \xi_{k}^{\prime}=\mu_{1}^{\prime}, \quad \widetilde{F}_{1}(z)=\int_{0}^{\infty} e^{-z x} \bar{F}_{1}(x) d x .
\end{gathered}
$$

The corresponding conditional moment generating functions of $\gamma^{+}(0)$ are given by

$$
\begin{aligned}
\widetilde{g}_{s}(z) & =\mathrm{E}\left[e^{-z \gamma^{+}(0)} / \xi^{+}\left(\theta_{s}\right)>0\right] \\
& =\frac{1}{1+b q_{-}(s) \widetilde{F}_{1}\left(\rho_{-}(s)\right)} \varphi_{1}(i z)+b q_{-}(s) \frac{z \widetilde{F}_{1}(z)-\rho_{-}(s) \widetilde{F}_{1}\left(\rho_{-}(s)\right)}{z-\rho_{-}(s)}
\end{aligned}
$$

for $s>0$. If $m<0$, then passing to the limit as $s \rightarrow 0$, one has

$$
\widetilde{g}_{0}(z)=\mathrm{E}\left[e^{-z \gamma^{+}(0)} / \xi^{+}>0\right]=\frac{\varphi_{1}(i z)+b \widetilde{F}_{1}(z)}{1+b \widetilde{F}_{1}(0)} .
$$

The moment generating function of $\xi^{+}\left(\theta_{s}\right)$ is determined by the prelimit generalization of the Pollaczek-Khinchin formula (6) with the moment generating function $\widetilde{g}_{s}(z)$ given by (27). The moment generating function of $\xi^{+}$is determined via the generalization of the Pollaczek-Khinchin formula (7) with the moment generating function $\widetilde{g}_{0}(z)$ given by (28), that is,

$$
\mathrm{E} e^{-z \xi^{+}}=\frac{p_{+}}{1-q_{+} \frac{\varphi_{1}(i z)+b \widetilde{F}_{1}(z)}{1+b \widetilde{F}_{1}(0)}}, \quad q_{+}=\frac{\lambda_{1}}{\lambda}\left(1+b \mu_{1}^{\prime}\right) .
$$

Proof. Since $1-z \widetilde{F}_{1}(z)=\varphi_{1}(i z)$, equality (25) follows from (24) after an integration by parts. This implies equality (26) for $m<0$ by passing to the limit as $s \rightarrow 0$. The conditional moment generating functions of $\gamma^{+}(0)$ written in (27) and (28) follow from relations (25) and (26). Those moment generating functions are used in the prelimit equality (6) and in the limit equality (7).

Theorem 1 and Corollaries 1 and 2 are proved under condition (3). This condition does not hold for almost lower semicontinuous processes with positive drift and for lower semicontinuous processes with unbounded variation. The second factorization equality (9) has no meaning in these cases, since

$$
f_{+}(s, z)=\mathrm{E}\left[e^{-z \gamma^{+}(0)-s \tau^{+}(0)} / \tau^{+}(0)<\infty\right]=1, \quad \mathrm{P}\left\{\gamma^{+}(0)=\tau^{+}(0)=0\right\}=1 .
$$

The characteristic functions of $\xi^{+}\left(\theta_{s}\right)$ and $\xi^{+}$can be found in a different way for these cases if $m<0$. 


\section{EVALUATION OF CHARACTERISTIC FUNCTIONS OF $\xi^{+}\left(\theta_{s}\right)$ AND $\xi^{+}$ FOR A GENERAL ALMOST LOWER SEMICONTINUOUS PROCESS}

Consider a process $\xi(t)=a t+\xi_{1}(t)+\xi_{2}(t)$ whose cumulant is

$$
\begin{gathered}
\psi(\alpha)=\psi_{1}(\alpha)+i \alpha a+\psi_{2}(\alpha), \quad \psi_{2}(\alpha)=-\frac{i \alpha \lambda_{2}}{b+i \alpha}, \quad \lambda_{2}>0, b>0, a \geq 0, \\
\psi_{1}(\alpha)=\int_{0}^{\infty}\left(e^{i \alpha x}-1\right) \Pi(d x), \quad \Pi(x)=\int_{x}^{\infty} \Pi(d y), \quad x>0, \\
\int_{0}^{1} x \Pi(d x)<\infty, \quad \Pi(0) \leq \infty .
\end{gathered}
$$

The variation of this process is bounded. Since the process is almost lower semicontinuous (see $\psi_{2}(\alpha)$ ), the Lundberg equation (13) has a negative root,

$$
r_{s}=-\rho_{-}(s)<0,
$$

and it determines the characteristic function of $\xi^{-}\left(\theta_{s}\right)$, namely

$$
\varphi_{-}(s, \alpha)=\frac{p_{-}(s)(b+i \alpha)}{\rho_{-}(s)+i \alpha}, \quad p_{-}(s)=\mathrm{P}\left\{\xi^{-}\left(\theta_{s}\right)=0\right\}=b^{-1} \rho_{-}(s) .
$$

An assertion on the distribution of $\xi^{+}\left(\theta_{s}\right)$ is proved in [4] (see relations (3.92)-(3.94) therein) by using the first (main) factorization equality (see Theorem 2.2 in [4).

Theorem 2. Let $\xi^{+}\left(\theta_{s}\right)$ be an almost lower semicontinuous process with bounded variation (see (30)). Then the characteristic function of $\xi^{+}\left(\theta_{s}\right)$ is given by the projective relations

$$
\begin{gathered}
\varphi_{+}(s, \alpha)=: \mathrm{E} e^{i \alpha \xi^{+}\left(\theta_{s}\right)}=\left[\frac{s b\left(\rho_{-}(s)+i \alpha\right)}{\rho_{-}(s)\left[s(b+i \alpha)-i \alpha\left(m b+\psi_{0}(\alpha)\right)\right]}\right]_{+}^{0}, \\
m=k^{\prime}(0)=a+k_{1}^{\prime}(0)-\lambda_{2} b^{-1}, \\
\psi_{0}(\alpha)=i \alpha a+\int_{0}^{\infty}\left(e^{i \alpha x}-1\right)(\Pi(d x)+b \Pi(x) d x), \\
{\left[c+\int_{-\infty}^{\infty} e^{i \alpha x} G(x) d x\right]_{+}^{0}=c+\int_{0}^{\infty} e^{i \alpha x} G(x) d x, \quad \int_{-\infty}^{\infty}|G(x)| d x<\infty .}
\end{gathered}
$$

If $m=\mathrm{E} \xi(1)<0$, then $\rho_{-}(s) \rightarrow 0$ as $s \rightarrow 0, \rho_{-}^{\prime}(0)=1 /|m|$, and the characteristic function of $\xi^{+}$is defined by

$$
\varphi_{+}(\alpha)=: \mathrm{E} e^{i \alpha \xi^{+}}=\frac{b|m|}{b|m|-\psi_{0}(\alpha)} .
$$

In other words,

$$
\begin{gathered}
\varphi_{+}(\alpha)=\frac{1}{1-\psi_{*}(\alpha)}, \\
\psi_{*}(\alpha)=i \alpha a^{*}+\int_{0}^{\infty}\left(e^{i \alpha x}-1\right) \Pi^{*}(d x), \quad a^{*}=\frac{a}{b|m|}, \\
\Pi^{*}(d x)=\frac{1}{b|m|}[\Pi(d x)+b \Pi(x) d x], \quad x>0 .
\end{gathered}
$$

Proof. The cumulant $\psi(\alpha)$ can be rewritten as follows:

$$
\psi(\alpha)=-i \alpha\left(b m+\psi_{0}(\alpha)\right)(b+i \alpha)^{-1} .
$$


According to equality (4),

$$
\varphi(s, \alpha)=\frac{s(b+i \alpha)}{s(b+i \alpha)-i \alpha\left(b m+\psi_{0}(\alpha)\right)} .
$$

Then

$$
\varphi(s, \alpha)=\varphi_{+}(s, \alpha) \varphi_{-}(s, \alpha)
$$

by the main factorization identity (see the last equation in (2.4) of [4]), and thus we derive from (31) and (35) that

$$
\varphi_{+}(s, \alpha)=\left[\varphi(s, \alpha) \frac{\rho_{-}(s)+i \alpha}{p_{-}(s)(b+i \alpha)}\right]_{+}^{0},
$$

whence (32) follows.

Passing to the limit as $s \rightarrow 0$ in (32) we prove for $m<0$ that

$$
\varphi_{+}(\alpha)=\frac{|m| b}{-\left(m b+\psi_{0}(\alpha)\right)}, \quad|m|=-m>0 .
$$

Note that the latter equality is similar to (33).

Relation (34) follows from (33) after simple algebra. The cumulant $\psi^{*}(\alpha)$ in (34) determines a nondecreasing process with the Lévy characteristics $a^{*}>0$ and $\Pi^{*}(d x)$, $x>0$. Relation (34) also implies that $\xi^{+} \doteq \xi^{*}\left(\theta_{1}^{\prime}\right)$, where $\mathrm{P}\left\{\theta_{1}^{\prime}>t\right\}=e^{-t}, t>0$.

If $a=0$ and $\Pi(0)=\lambda_{1}<\infty$, then $m=\lambda_{1} \mu_{1}^{\prime}-\lambda_{2} b^{-1}, b|m|=\lambda_{2}-b \lambda_{1} \mu_{1}^{\prime}$, and

$$
\psi_{0}(\alpha)=\lambda_{1} \int_{0}^{\infty} e^{i \alpha x}\left(F_{1}(d x)+b \bar{F}_{1}(x) d x\right)-\lambda_{1}\left(1+b \mu_{1}^{\prime}\right) .
$$

Substituting the expression for $b|m|$ and $\psi_{0}(\alpha)$ in (33), we see that equalities (29) and (33) are equivalent.

\section{EVALUATION OF CHARACTERISTIC FUNCTIONS OF $\xi^{+}\left(\theta_{s}\right)$ AND $\xi^{+}$ FOR A LOWER SEMICONTINUOUS PROCESS}

The characteristic function of a lower semicontinuous process $\xi(t)$ is determined by the cumulant

$$
\psi(\alpha)=i \alpha a-\frac{\sigma^{2}}{2} \alpha^{2}+\int_{0}^{\infty}\left(e^{i \alpha x}-1-\mathbb{1}_{x \leq 1} i \alpha x\right) \Pi(d x)
$$

$\left(a<0\right.$ if $\left.\sigma=0, \int_{0}^{1} x^{2} \Pi(d x)<\infty\right)$.

The following relation,

$$
\begin{gathered}
k(s, \alpha)=\int_{0}^{\infty} e^{i \alpha x} \int_{-\infty}^{0} \Pi(x-y) d P_{-}(s, y) d x, \quad P_{-}(s, x)=\mathrm{P}\left\{\xi^{-}\left(\theta_{s}\right)<x\right\}, \quad x<0, \\
C_{*}(s)= \begin{cases}(2 s)^{-1} \sigma^{2} P_{-}^{\prime}(s, x), & \sigma>0, \\
s^{-1} p_{-}(s)[a]^{+}, & \sigma=0,[a]^{+}=\max (0, a),\end{cases}
\end{gathered}
$$

can be proved for an arbitrary homogeneous process with independent increments by using Corollary 2.2 in [4] (or Corollary 1 in [5]; see (14) therein). If the cumulant of $\xi(t)$ is given by (36) $(a<0$ if $\sigma=0)$, then

$$
C_{*}(s)=\frac{\sigma^{2}}{2 s} \rho_{-}(s) .
$$


If $m<0$ and $\sigma>0$, then

$$
\begin{gathered}
C_{*}(0)=\frac{\sigma^{2}}{2}|m|, \\
P_{-}(s, x)=e^{\rho_{-}(s) x}, \quad x \leq 0, \quad \sigma \geq 0, \\
k(s, \alpha)=\frac{\rho_{-}(s)}{\rho_{-}(s)+i \alpha} \int_{0}^{\infty}\left(e^{i \alpha x}-e^{-\rho_{-}(s) x}\right) \Pi(x) d x,
\end{gathered}
$$

$C_{*}(s)=0$ if $\sigma=0$.

If $m<0$, then

$$
\rho_{-}^{\prime}(0)=|m|^{-1}
$$

and

$$
\begin{gathered}
k^{\prime}(0, \alpha)=\frac{1}{i \alpha|m|} \int_{0}^{\infty}\left(e^{i \alpha x}-1\right) \Pi(x) d x, \\
\int_{0}^{1} x \Pi(x) d x=\int_{0}^{1} x^{2} \Pi(d x)<\infty .
\end{gathered}
$$

The following result can be proved by using relations (36) for $C_{*}(s)$ and $k(s, \alpha)$ if the process is lower semicontinuous.

Theorem 3. If $\xi(t)$ is a process whose cumulant is given by (36) and $\sigma \geq 0$ ( $a<0$ if $\sigma=0)$, then

$$
\begin{aligned}
\varphi_{+}(s, \alpha)=\left\{1-i \alpha\left[\frac{\sigma^{2} \rho_{-}(s)}{2 s}\right.\right. & \\
& \left.\left.\quad+\frac{\rho_{-}(s)}{s} \frac{1}{\rho_{-}(s)+i \alpha} \int_{0}^{\infty}\left(e^{i \alpha x}-e^{-\rho_{-}(s) x}\right) \Pi(x) d x\right]\right\}^{-1} .
\end{aligned}
$$

If $m<0$, then

$$
\varphi_{+}(\alpha)=\left\{1-i \alpha \frac{\sigma^{2}}{2}|m|^{-1}-|m|^{-1} \int_{0}^{\infty}\left(e^{i \alpha x}-1\right) \Pi(x) d x\right\}^{-1}=\frac{1}{1-\psi_{*}(\alpha)},
$$

where

$$
\begin{gathered}
\psi_{*}(\alpha)=i \alpha a_{*}+\int_{0}^{\infty}\left(e^{i \alpha x}-1\right) \Pi_{*}(d x), \\
a_{*}=\frac{\sigma^{2}}{2}|m|^{-1}, \quad \Pi_{*}(d x)=|m|^{-1} \Pi(x) d x, \quad x>0 .
\end{gathered}
$$

The cumulant $\psi_{*}(\alpha)$ determines a nondecreasing process $\xi_{*}(t)$ with a bounded variation

$$
\mathrm{E} e^{i \alpha \xi^{+}}=\mathrm{E} e^{\xi_{*}\left(\theta_{1}^{\prime}\right)}
$$

where $\theta_{1}^{\prime}$ is an exponential random variable with parameter 1.

Proof. Substituting (38) in (36) and (40) in (39) we obtain (39) by passing to the limit as $s \rightarrow 0$, since

$$
\lim _{s \rightarrow 0} \frac{\rho_{-}(s)}{s}=\frac{1}{|m|} \quad \text { for } m<0 .
$$

Note that assumption (3) (meaning that $p_{+}(s)>0$ ) is not used in the proofs of Theorems 2 and 3. If, nevertheless, this condition holds, then the corresponding relations imply the equalities similar to the prelimit or limit Pollaczek-Khinchin formula. In particular, if $m<0$, then

$$
\Pi(0)=\lambda_{1}<\infty, \quad \sigma=0
$$


(do not miss $\Pi(0)$ with $\left.\widetilde{\Pi}(0)=\int_{0}^{\infty} \Pi(x) d x\right)$. Equality (40) can easily be reduced to the classical Pollaczek-Khinchin formula (21). Finally, equality (40) implies that

$$
\xi^{+} \doteq \xi_{*}\left(\theta_{1}^{\prime}\right)
$$

\section{BIBLIOGRAPHY}

1. A. A. Borovkov, Stochastic Processes in Queueing Theory, Nauka, Moscow, 1972; English transl., Springer-Verlag, New York-Heidelberg-Berlin, 1976. MR0315800 (47:4349) MR.0391297 (52:12118)

2. G. P. Klimov, Stochastic Queueing Systems, Nauka, Moscow, 1966. (Russian) MR0207064 $(34: 6880)$

3. S. Asmussen, Ruin Probabilities, World Scientific, Singapore, 2000. MR.1794582 (2001m:62119)

4. D. V. Gusak, Limit problems for processes with independent increments in the risk theory, Proceedings of the Institute of Mathematics, National Academy of Science of Ukraine, vol. 67, Kiev, 2007. (Ukrainian)

5. D. V. Gusak, Distribution of overjump functionals of semicontinuous homogeneous processes with independent increments, Ukrain. Matem. Zh. 54, no. 3, 303-322; English transl. in Ukr. Math. J. 54 (2002), no. 3, 371-379. MR.1952790(2003j:60067)

6. A. V. Skorokhod, Random Processes with Independent Increments, Nauka, Moscow, 1964; English transl., Kluwer Academic Publishers, Dordrecht, 1991. MR.1155400 (93a:60114)

252601, Institute of Mathematics, National Academy of Sciences of Ukraine, TereShChenkivs'Ka Street, 3, Kiev 01004, Ukraine

E-mail address: random@imath.kiev.ua

Received 27/FEB/2009

Translated by S. V. KVASKO 